<smiles>C1=C2C=C12</smiles> 


\title{
Franz Kafka
}

\author{
Internationale International \\ Bibliographie Bibliography \\ der Primär- und of Primary and \\ Sekundärliteratur Secondary Literature
}

\author{
Band I Volume I \\ Bibliographie der Primärliteratur Bibliography of Primary Literature \\ $1908-1997 \quad 1908-1997$ \\ Band II Volume II \\ Kommentierte Bibliographie der \\ Annotated Bibliography of \\ Sekundärliteratur 1955-1997 Secondary Literature 1955-1997 \\ Teil 1: 1955-1980 Part 1: 1955-1980 \\ Teil 2: 1981-1997 Part 2: 1981-1997 \\ mit Nachträgen zu Teil 1 with Addenda to Part 1
}




\section{Maria Luise Caputo-Mayr \\ Julius Michael Herz}

\section{Franz Kafka}

Internationale International

Bibliographie Bibliography

der Primär- und of Primary and

Sekundärliteratur Secondary Literature

Eine Einführung An Introduction

2., erweiterte und $\quad 2^{\text {nd }}$, enlarged and überarbeitete Auflage revised edition

\section{Band II Volume II}

Bibliographie der Bibliography of Sekundärliteratur Secondary Literature $1955-1997 \quad 1955-1997$

Teil 1: 1955 - $1980 \quad$ Part 1: $1955-1980$ 
Gedruckt mit Unterstützung des / Printed with support of

Förderungs- und Beihilfefonds Wissenschaft der VG-Wort GmbH, München

Dank für die finanzielle Unterstützung ergeht außerdem an folgende Institutionen /

The printing of these volumes was made possible by the following institutions:

Deutsche Schillergesellschaft, Deutsches Literaturarchiv in Marbach/Neckar,

Generalkonsulat der Bundesrepublik Deutschland in New York,

Deutscher Akademischer Austauschdienst (DAAD),

Österreichisches Bundesministerium für auswärtige Angelegenheiten und

Bundesministerium für Bildung, Wissenschaft und Kultur, Wien.

Die Deutsche Bibliothek - CIP-Einheitsaufnahme

Caputo-Mayr, Maria Luise:

Franz Kafka : internationale Bibliographie

der Primär- und Sekundärliteratur = Franz Kafka $/$

Maria Luise Caputo-Mayr ; Julius Michael Herz. - München : Saur

ISBN 3-907820-97-5

Bd. 2. Kommentierte Bibliographie der Sekundärliteratur 1955 - 1997.

Teil 1. 1955 - 1980. - 2., erw. und überarb. Aufl.. - 2000

ISBN 3-907820-65-7

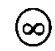

Gedruckt auf säurefreiem Papier / Printed on acid free paper

(C) 2000 by K. G. Saur Verlag GmbH \& Co. KG, München

Printed in the Federal Republic of Germany

Alle Rechte vorbehalten / All Rights Strictly Reserved

Jede Art der Vervielfältigung ohne Erlaubnis des Verlags ist unzulässig

Druck und Binden / Printed and bound by:

Strauss Offsetdruck GmbH, Mörlenbach

ISBN 3-907820-65-7 (Band II/Teile 1 und 2) 